\title{
A CoMparative STUdy Of DifFerent QUEUING TECHNIQUES IN VOIP, VIDEO CONFERENCING AND FILE TRANSFER
}

\author{
Mohammad Mirza Golam Rashed and Mamun Kabir \\ Department of ETE, Daffodil International University \\ E-Mail: mgrashed@daffodilvarsity.edu.bd, mamunkabirbd@yahoo.com
}

\begin{abstract}
Today's Internet only provides Best Effort Service. Traffic is processed as quickly as possible, but there is no guarantee of timelines or actual delivery. With the rapid transformation of the Internet into a commercial infrastructure, demands for service quality have rapidly developed. People of the modern world are very much dependent on various network services like VOIP, Videoconferencing and File Transfer. Different types of Traffic Management systems are used in those services. Queuing is one of the very vital mechanisms in traffic management system. Each router in the network must implement some queuing discipline that governs how packets are buffered while waiting to be transmitted. This paper gives a comparative analysis of three queuing systems FIFO, PQ and WFQ. The study has been carried out on some issues like: Traffic dropped Traffic Received and packet end to end delay and the simulation results shows that WFQ technique has a superior quality than the other techniques.
\end{abstract}

Keywords: IP QoS, FIFO, PQ, FQ, WFQ

\section{Introduction}

The current Internet is based on IP protocol and supports only best effort services. With the exponential growth of Internet during the last years, IP networks are expected to support not only typical services like ftp and email, but also real-time services and video streaming application. The traffic characteristics of these applications require a certain Quality of Service (QoS) from the network in terms of bandwidth and delay requirements [1].

The biggest problems in a network are related to the allocation of network resources, as buffers and link band width, to different users. A limited amount of resources has to be shared among many different competing traffic flows in an efficient way in order to maximize the performance and the use of the network resources. The behavior of routers in terms of packet handling can be controlled to achieve different kind of services [1]. This paper demonstrates the performance of a number of packet handling mechanisms and produces a comparative picture of them using the simulation software OPNET.

\section{Packet Handling Techniques}

Various queuing disciplines can be used to control which packets get transmitted and which packets which packets get dropped. The queuing disciplines are:

1. First-in-first-out (FIFO) queuing.

2. Priority queuing(PQ)

3. Weighted-Fair queuing.(WFQ)

FIFO is an acronym for First In First Out .This expression describes the principle of a queue or first-come first serve behavior: what comes in first is handled first, what comes in next waits until the first is finished etc. Thus it is analogous to the behavior of persons "standing in a line" or "Queue" where the persons leave the queue in the order they arrive. First In First Out (FIFO) is the most basic queuing discipline. In FIFO queuing all packets are treated equally by placing them into a single queue, then servicing them in the same order they were placed in the queue. FIFO queuing is also referred to as First Come First Serve (FCFS) queuing [2].

Priority Queuing assigns multiple queues to a network interface with each queue being given a priority level. A queue with higher priority is processed earlier than a queue with lower priority. Priority Queuing has four preconfigured queues, high medium, normal and low priority queue. By default each of these queues has 20,40, 60 and 80 packets capacity [3].

If packets arrive in the high queue then priority queuing drops everything its doing in 
order to transmit those packets, and the packets in other queue is again empty. When a packet is sent out an interface, the priority queues on that interface are scanned for packets in descending order for priority. The high priority queue is scanned first, then the medium priority queue and then so on.The packet at the head of the highest queue is chosen for transmission. This procedure is repeated every time when a packet is to be sent. The maximum length of a queue is defined by the length limit. When a queue is longer the limit packets are dropped [4].

In QoS, a flow-based queuing algorithm that schedules low-volume traffic first, while letting high-volume traffic share the remaining bandwidth. This is handled by assigning a weight to each flow, where lower weights are the first to be serviced [5]. WFQ is a generalization of fair queuing (FQ). Both in WFQ and FQ, each data flow has a separate FIFO queue. In FQ, with a link data rate of $\mathrm{R}$, at any given time the $\mathrm{N}$ active data flows (the ones with non-empty queues) are serviced simultaneously, each at an average data rate of R / N. Since each data flow has its own queue, an ill-behaved flow (who has sent larger packets or more packets per second than the others since it became active) will only punish itself and not other sessions [6].

Here different queuing discipline in the routers can affect the performance of the applications and the utilization of the network resources.

\section{Network Design and Configuration}

To execute all the experimental works the following network design has been taken into consideration [7]. At the first step single traffic is used for each of the functions such as Ftp, Video Conferencing and VOIP which is shown in Fig. 1.

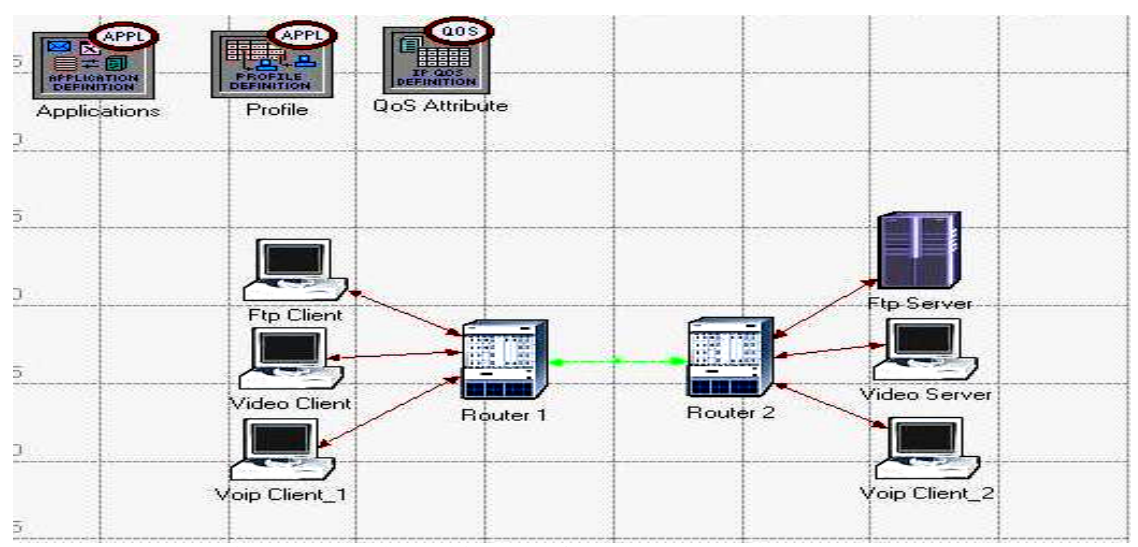

Fig. 1 Network Architecture for FIFO, PQ and WFQ

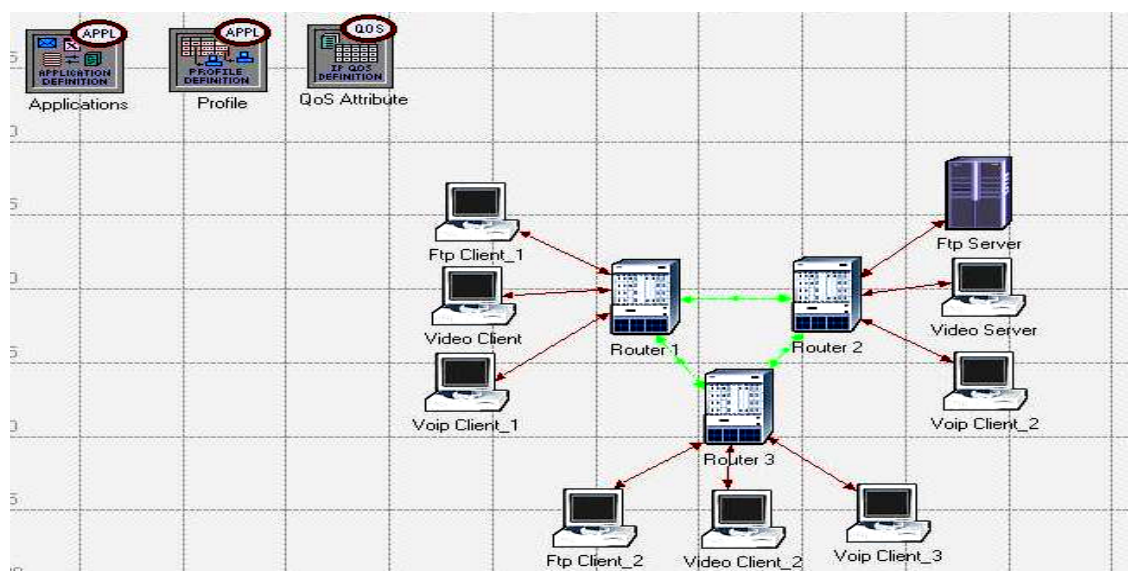

Fig. 2 Network Architecture for FIFO1, PQ1 and WFQ1 


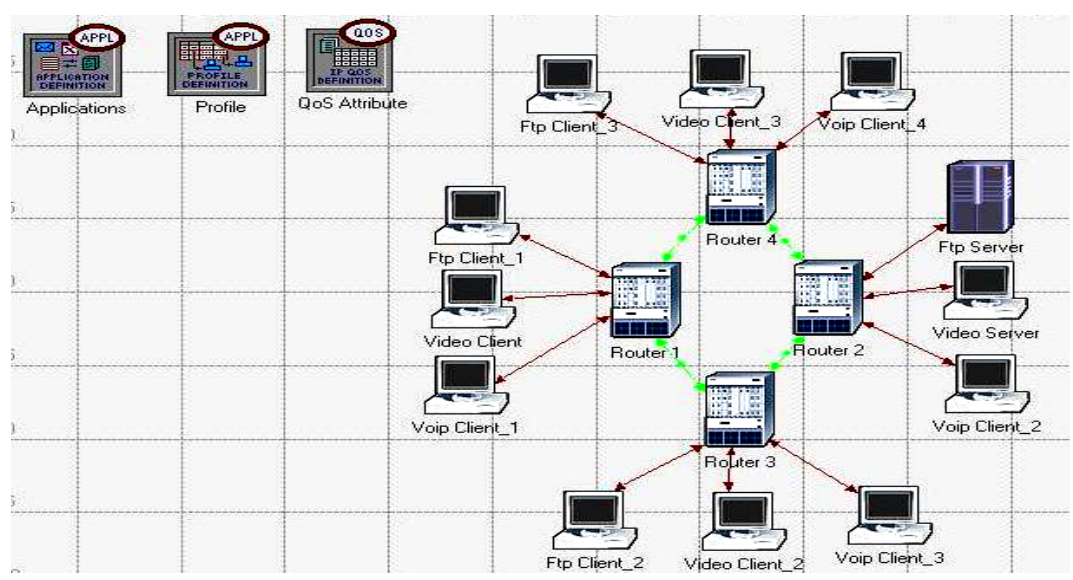

Fig. 3 Network Architecture for FIFO2, PQ2 and WFQ2

The basic architecture given in Fig. 3 has been modified in two steps to consider the increment of traffic volume. They are given in the figures Fig.2 and Fig. 3. Simulations for the three techniques FIFO, PQ and WFQ are executed for the three networks of Figs.13 respectively.

\section{Configuration}

The bellow configurations applied in the Opnet Modeler and simulated to get results. 1. The routers are connected with PPP_DS1link.

2. The Work stations and the servers are connected with routers with 10Base_T links.
3. In the field of FTP application "High Load" has been selected, Constant (10) to Inter-Request Time and Constant (1000000) to File Size are assigned.

4. In the field of Video Application "Low Resolution Video" has been selected for Video Conferencing, Streaming Multimedia (4) to ToS is assigned.

5. In field of VoIP application PCM Quality Speech to Voice and Interactive Voice (6) to ToS is assigned.

6. Different queuing discipline in the routers can affect the performance of the applications and the utilization of the network resources. So routers need to be configured for those three Queuing disciplines. The configurations are given Figs. 4 (a)-(c).

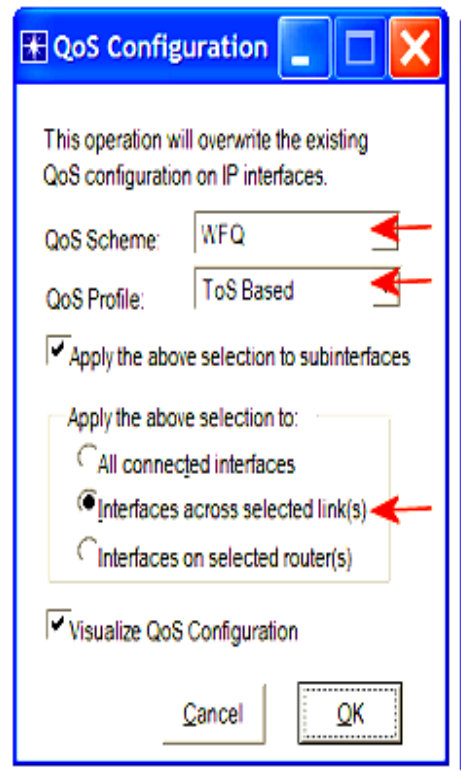

Fig. 4 (a) Configuration for FIFO

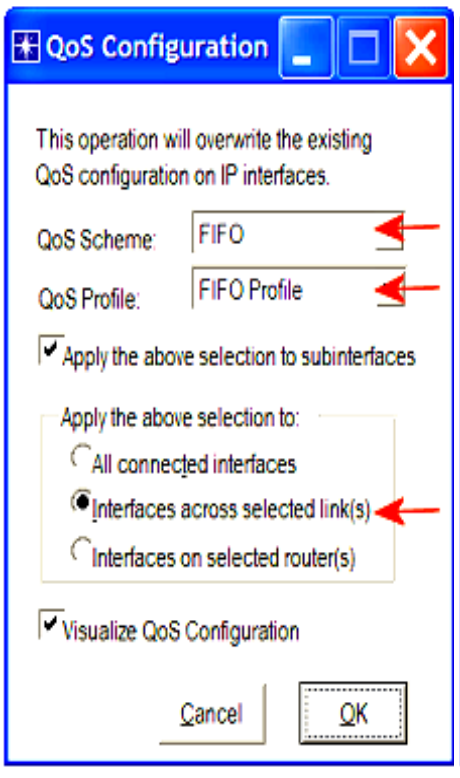

Router Configuration

(b) configuration for PQ

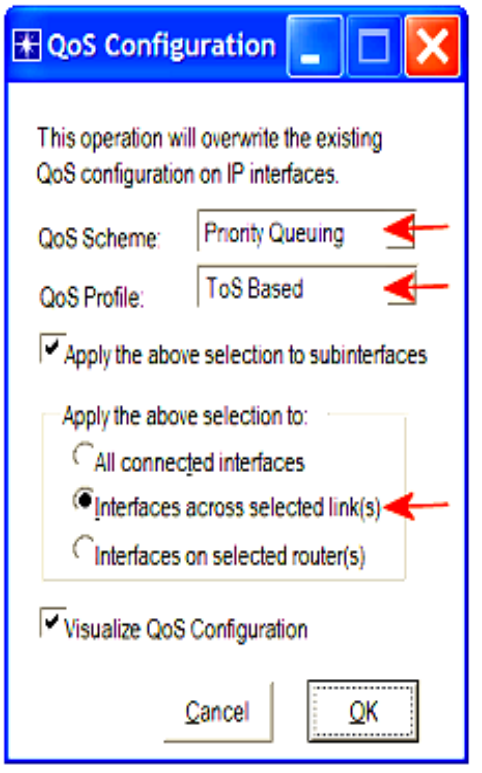

(c) configuration for WFQ 
5 Simulation and Analysis

5.1 Traffic dropping: Three simulations have been executed using OPNET software for every queuing scheme in terms of packet dropping, traffic receiving, packet end-to-end delay etc and it is tested for Video Conferencing, Voice Traffic and FTP.

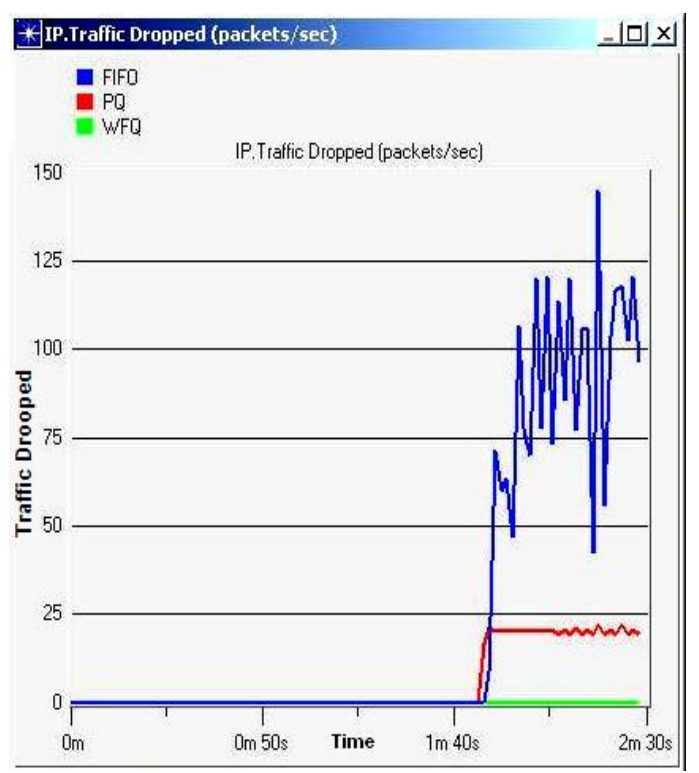

Fig. 5 (a). Traffic drop for FIFO, PQ and WFQ

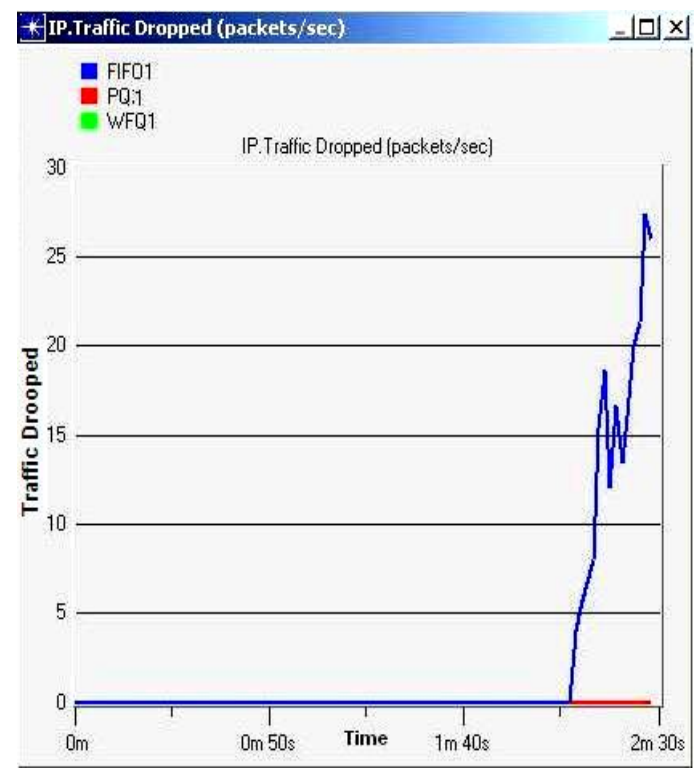

Fig. 5 (b). Traffic drop for FIFO1, PQ1 and WFQ1

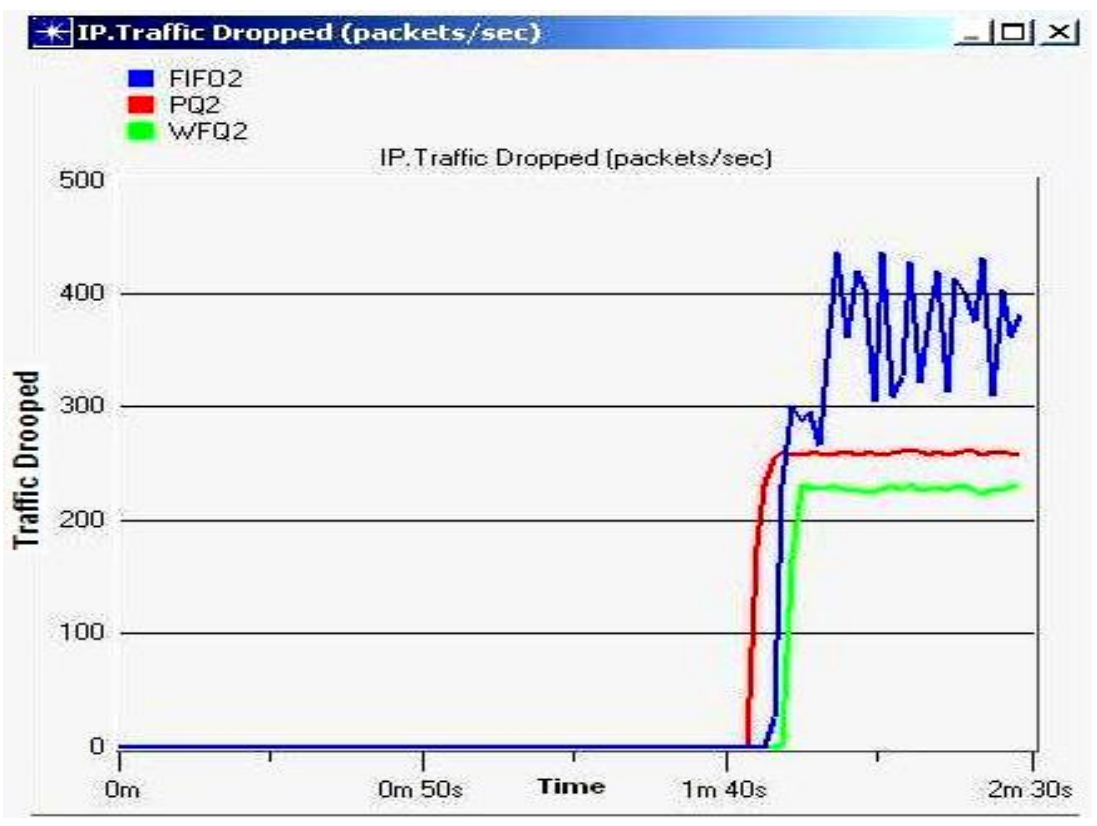

Fig. 5 (c) Traffic drop for FIFO2, PQ2 and WFQ2

Figures (Fig.: 5a, 5b, 5c) shows traffic dropping statistics, where it can be observed that in the cases of FIFO, PQ, WFQ(Fig 5a) the packet drop starts at near 95 sec. Packet drop for FIFO is higher, PQ is lower and for WFQ there is no packet drop. In case of FIFO1, PQ1, WFQ1(Fig 5b) the packet drop starts at near 115sec. packet drop here for FIFO1 is higher, there is no packet drop for PQ1 and WFQ1. In case of FIFO2, PQ2, WFQ2(Fig 5c) the packet drop starts at near 95 sec. Packet drop for FIFO in this case is higher, PQ is semi lower and WFQ is lower. 


\subsection{Traffic Received}

\subsubsection{Traffic Received: (For VOIP)}

Figures (Fig.: 6a, 6b, 6c) shows traffic Received statistics for VoIP, where it can be observed that as the traffic increased the

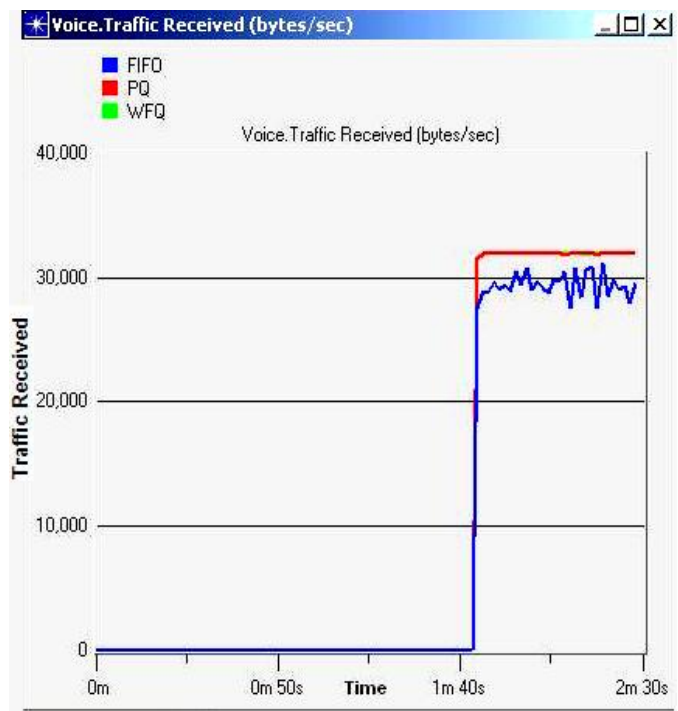

Fig. 6 (a) Traffic Received for FIFO, PQ \& WFQ performance graph line increased in both group of FIFO and PQ. The performance graph line of FIFO group is always lowered compared to the groups PQ and WFQ.

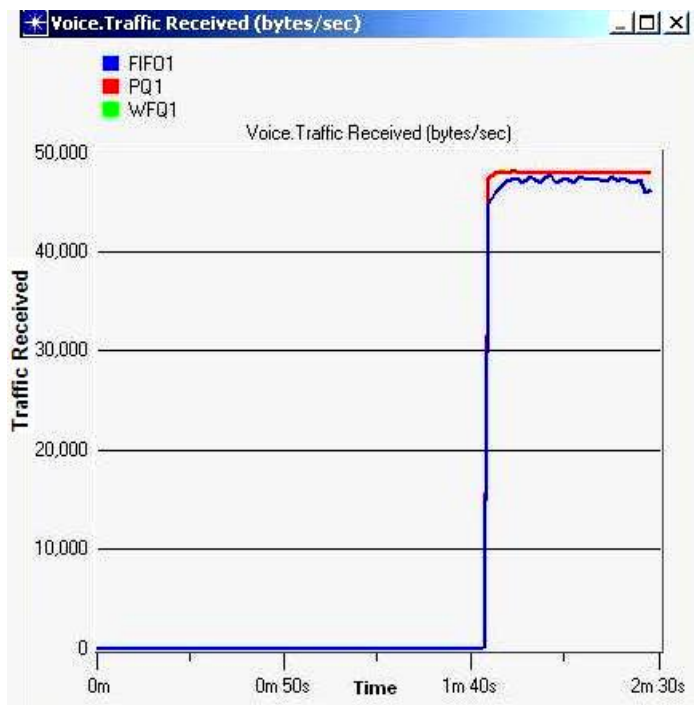

Fig. 6 (b) Traffic Received for FIFO1, PQ1 \& WFQ1

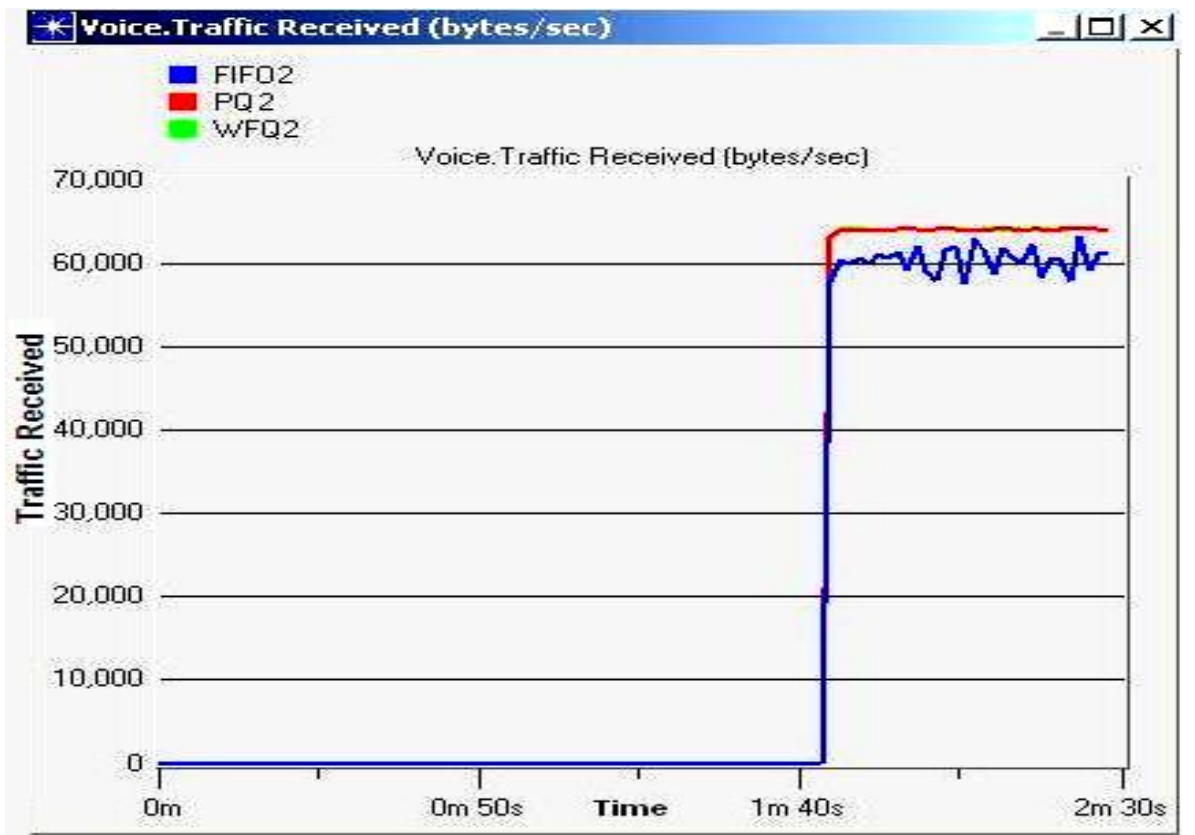

Fig. 6 (c) Traffic Received for IFO2, PQ2 and WFQ2

\subsubsection{Traffic Received: (For Video} conferencing)

Figures (Fig.: 7a, 7b, 7c) shows traffic Received statistics for Video conferencing, where it can be observed that in cases of FIFO, PQ, WFQ and FIFO2, PQ2,_WFQ2 video receiving rate graph WPQ group is always higher than the performance graph of FIFO group and PQ group is lower than the other two groups.. In case of FIFO1, PQ1, WFQ1 the performance graph of PQ1 is even at the time being and both FIFO1 and WFQ1 is near same. 

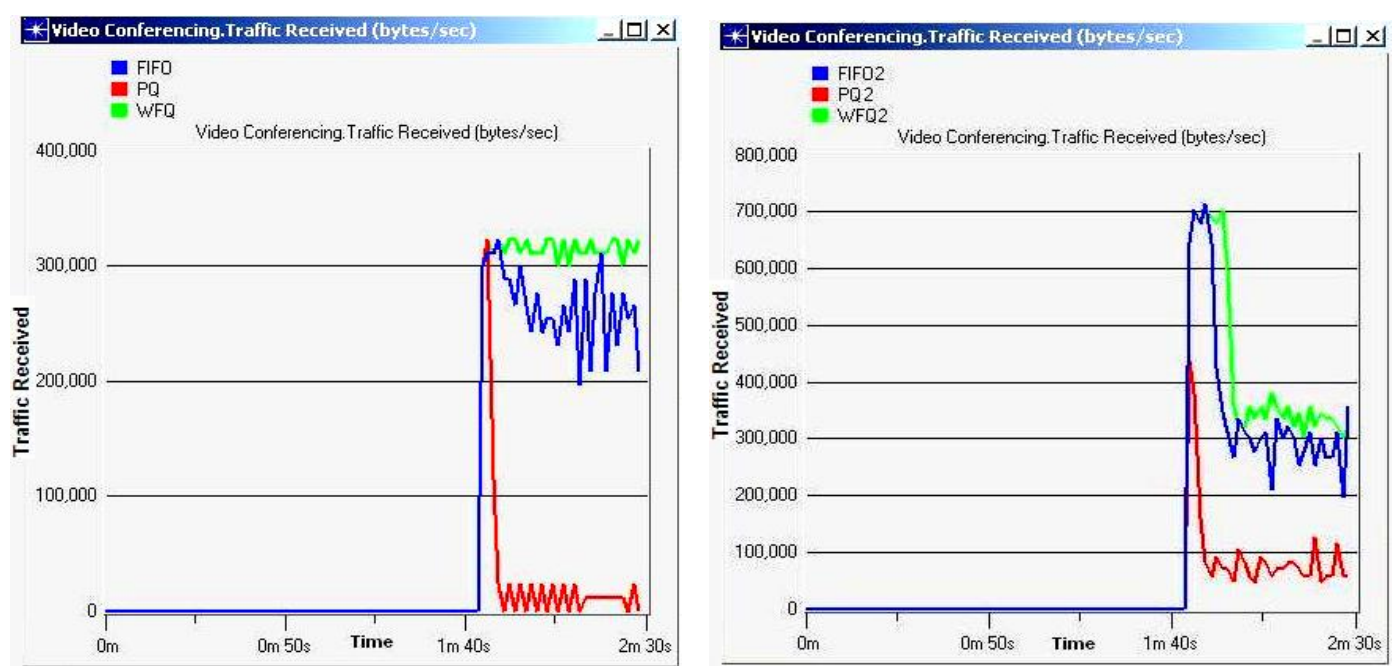

Fig. 7 (a) Traffic Received for FIFO, PQ \& WFQ

Fig. 7 (b) Traffic Received for FIFO1, PQ1\& WFQ1

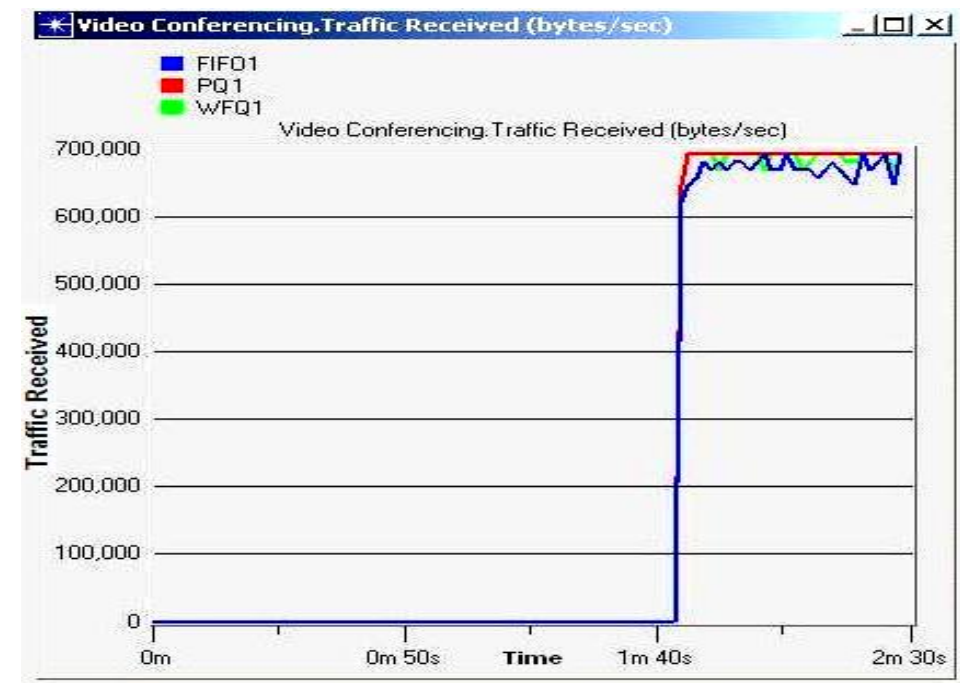

Fig. 7 (c) Traffic Received for FIFO2, PQ2 and WFQ2

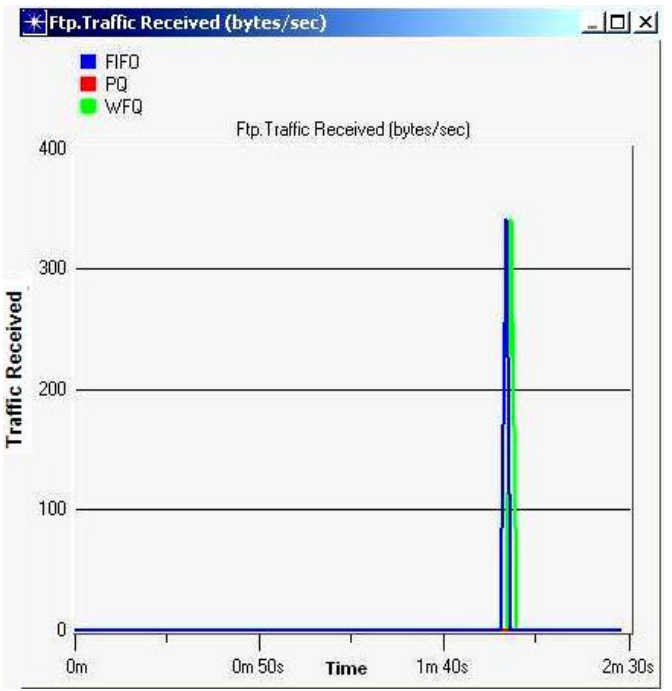

Fig. 8 (a) Traffic Received for FIFO, PQ \& WFQ

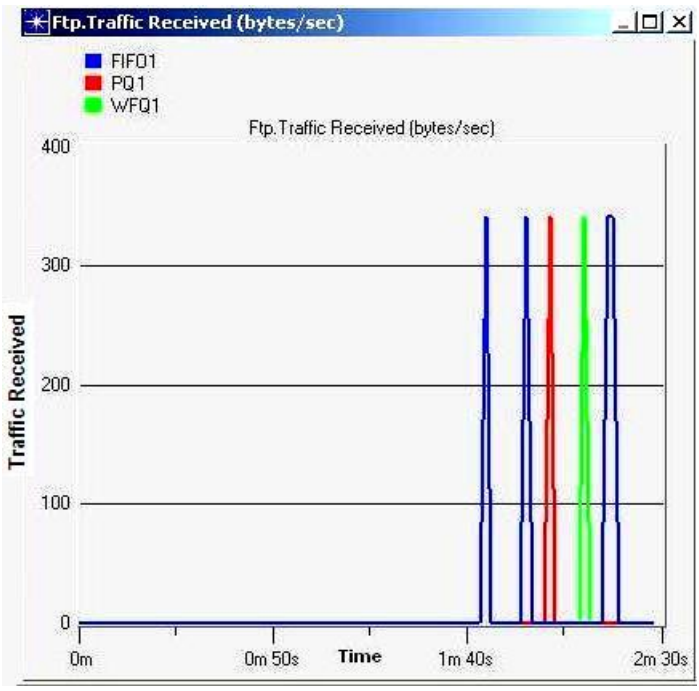

Fig. 8 (b) Traffic Received for FIFO1, PQ1 \&WFQ1 


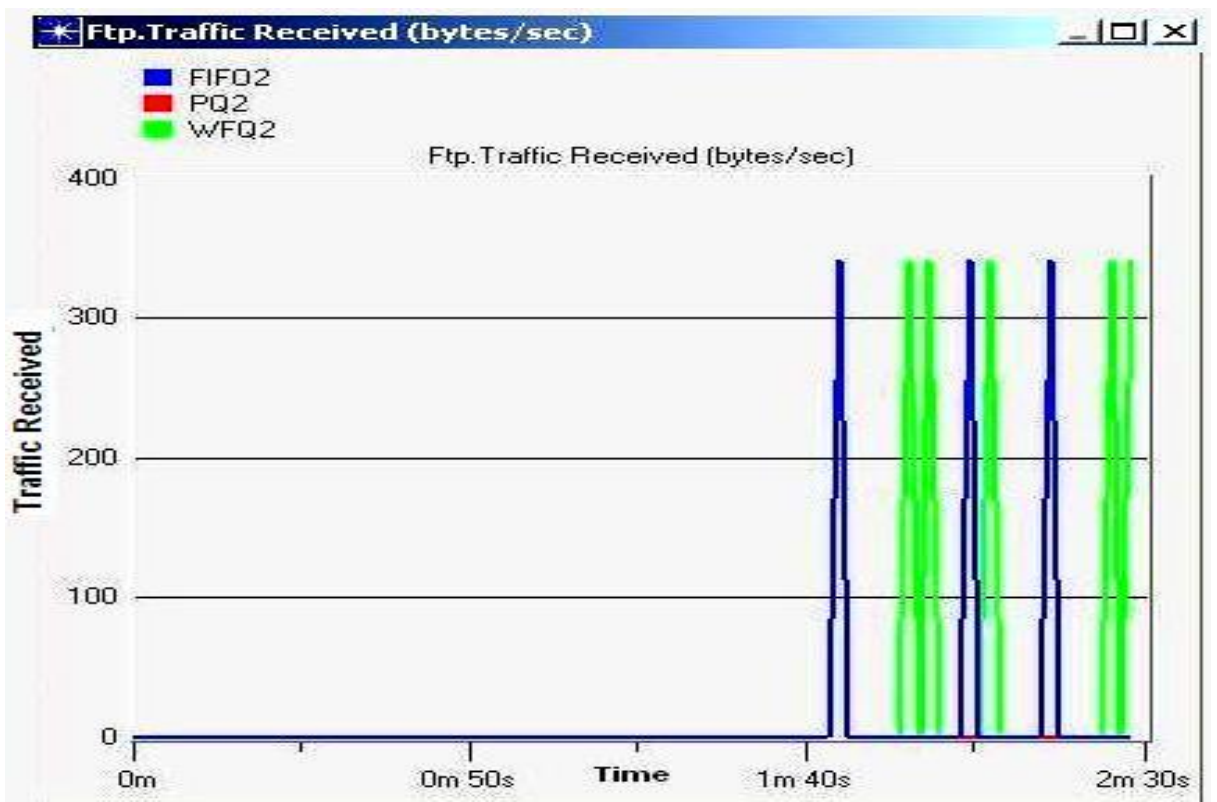

Fig. 8 (c) Traffic Received for FIFO2, PQ2 and WFQ2

\subsubsection{Traffic Received: (For FTP)}

Figures (Fig.: 8a, 8b, 8c) shows traffic Received statistics for FTP, where it can be observed that as the traffic increased the file receiving performance graph line is same in both group of FIFO and WFQ.The performance graph line of $\mathrm{PQ}$ is only same in case of FIFO1, PQ1, and WFQ1 and in case of FIFO, PQ, WFQ and FIFO2, PQ2, WFQ2 PQ's file receiving rate is almost zero.

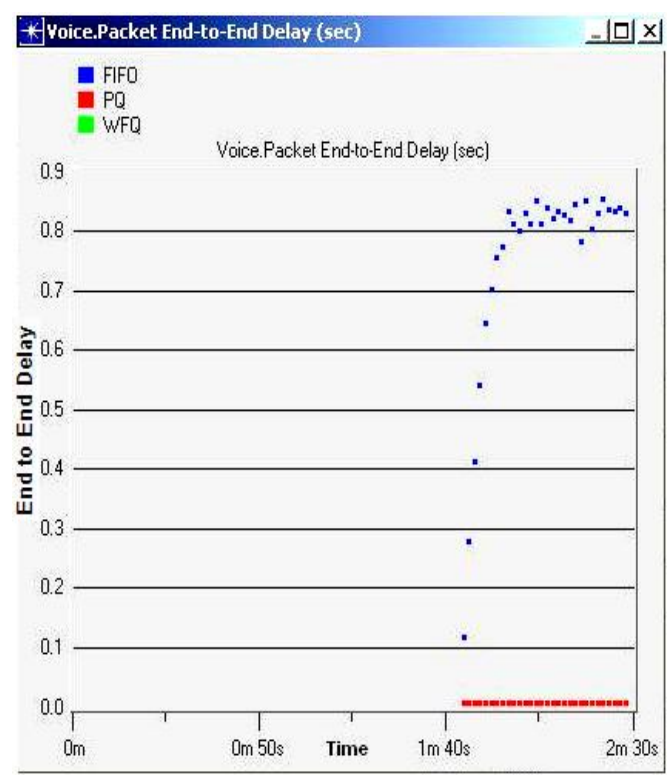

Fig. 9 (a) End to End delay for FIFO, PQ \& WFQ

\subsection{Packet end to end delay}

5.3.1 Packet end to end delay: (For VOIP)

Figures (Fig.: 9a, 9b, 9c) shows Packet end to end delay time for VoIP. For both the cases such as time increase or traffic increase PQ and WFQ groups packet end to end delay line always shows the same characteristics that is packet end to end time delay is nearly zero. FIFO group is always higher.

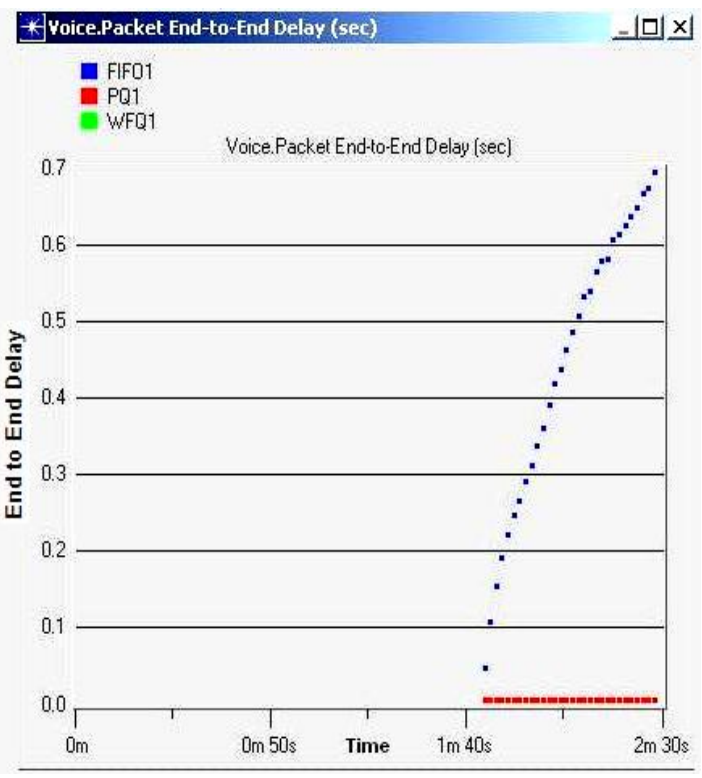

Fig. 9 (b) End to End delay for FIFO1, PQ1\& WFQ1 


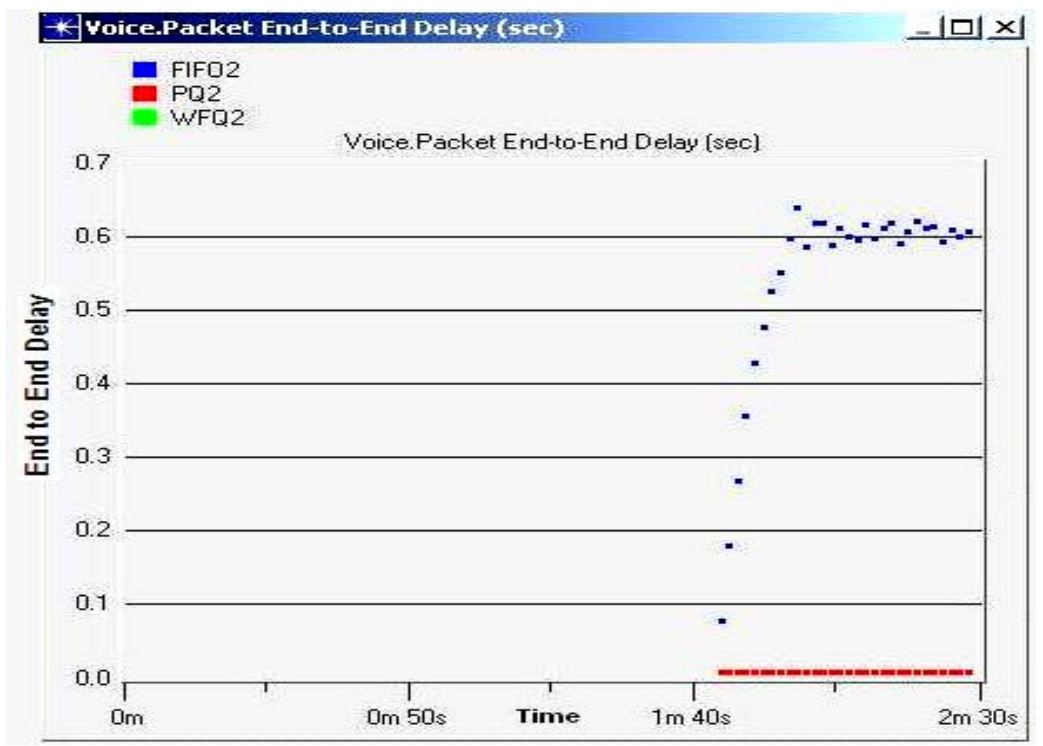

Fig. 9 (c) End to End delay for FIFO2, PQ2 \& WFQ2

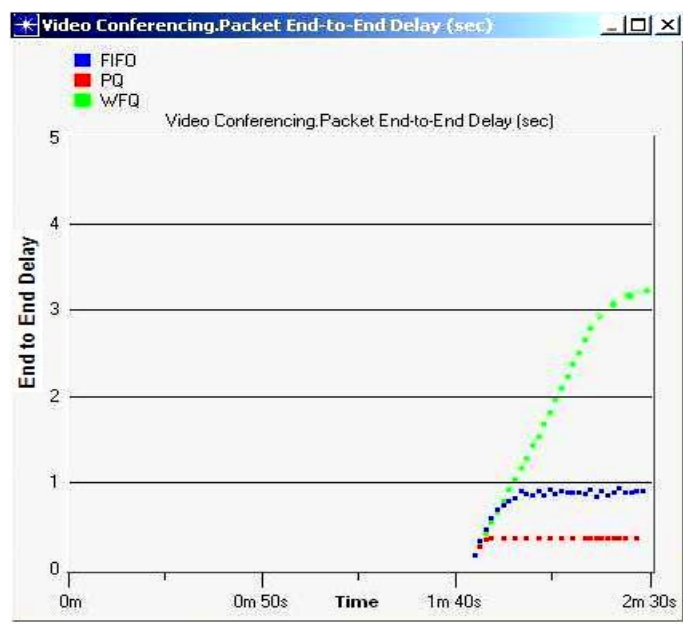

Fig. 10 (a) End to End delay for FIFO, PQ \& WFQ

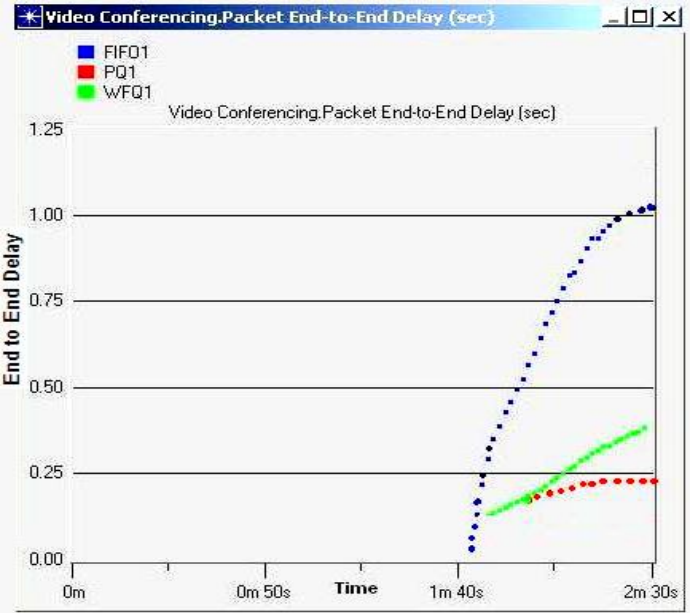

Fig. 10 (b) End to End delay for FIFO1, PQ1\& WFQ1

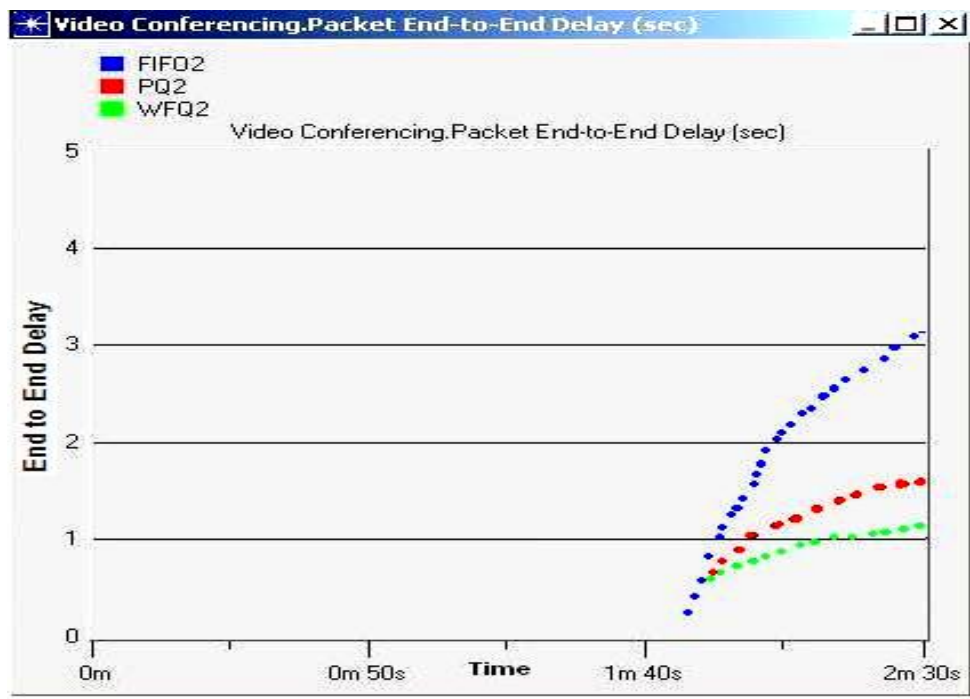

Fig. 10 (c) End to End delay for FIFO2, PQ2 and WFQ2 


\subsubsection{Packet end to end delay: (For Video conferencing)}

Figures 10a, 10b and 10c shows Packet end to end delay time for Video conference, where it can be observed that as the traffic increased the Packet end to end delay time is decreasing for WFQ groups when Packet end to end delay time is increasing for PQ groups. Like VoIP, FIFO group's Packet end to end delay time is also always higher in Video conference.

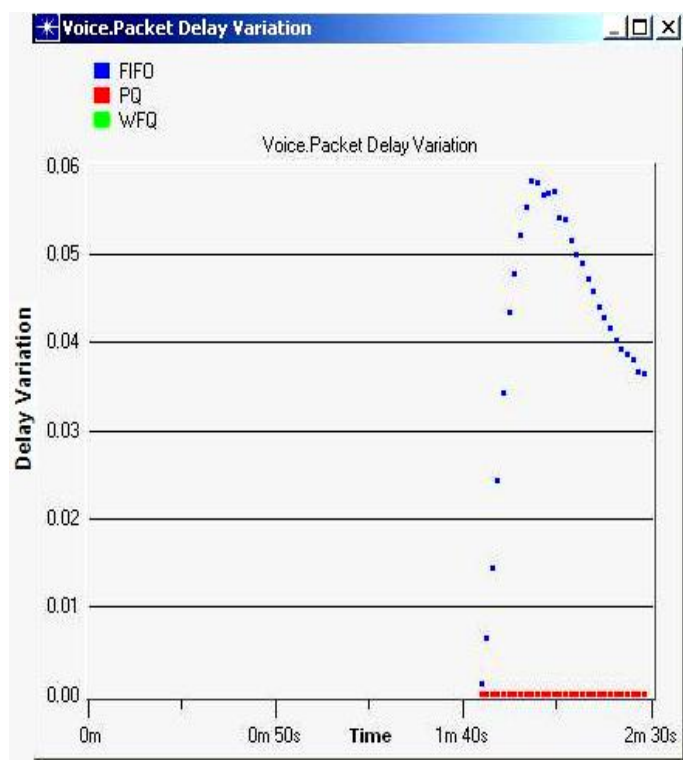

Fig. 11 (a) Delay variation for FIFO, PQ \& WFQ

\subsection{Packet delay variations}

5.4.1 Packet delay variations: (For VOIP)

Figures 11a, 11b and 11c shows Packet delay variation time for VoIP, for all the cases such as time increase or traffic increase PQ and WFQ groups packet delay time line always shows the same characteristics that is packet delay time is nearly zero and for FIFO group it is always higher.

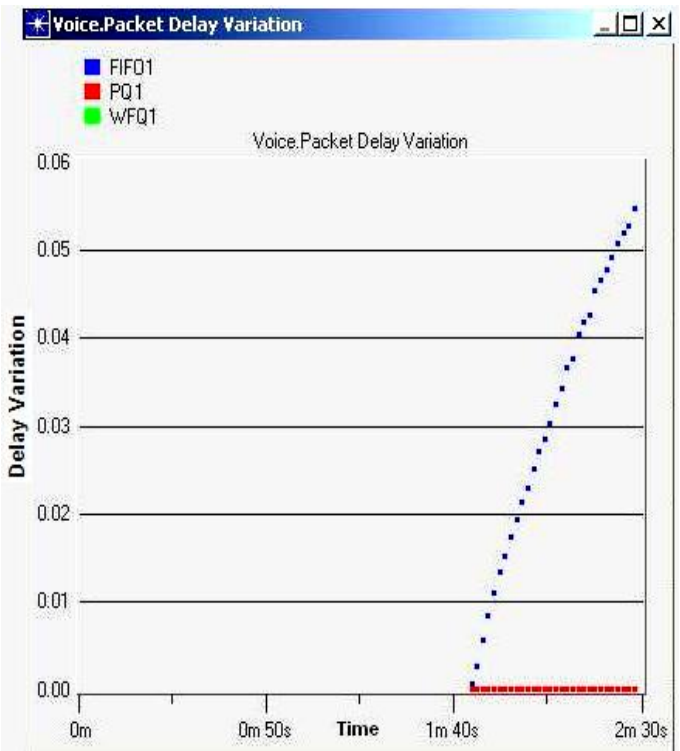

Fig. 11 (b) Delay variation forFIFO1, PQ1\& WFQ1

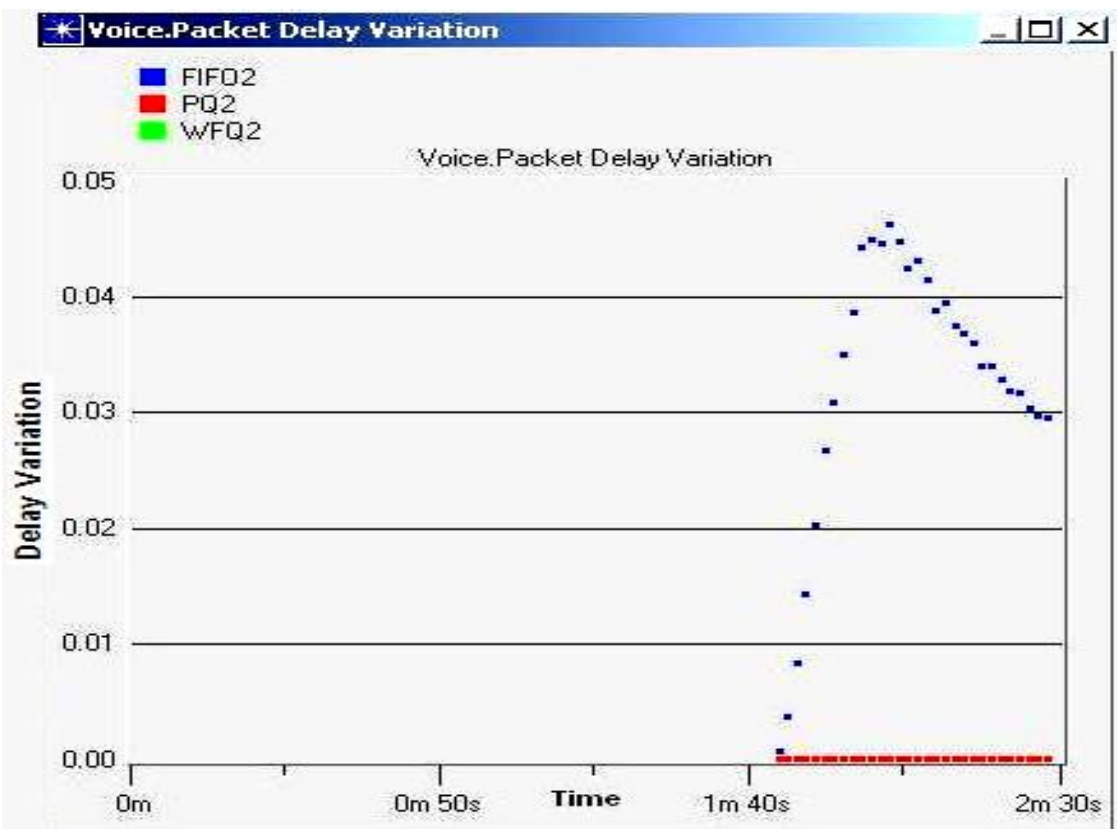

Fig. 11 (c) Delay variation for FIFO2, PQ2 and WFQ2 


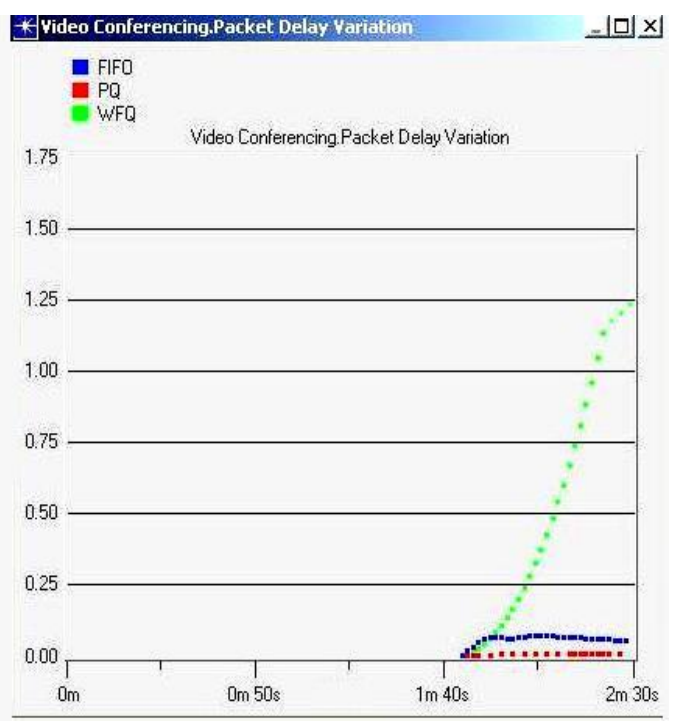

Fig. 12 (a) Delay variation for FIFO, PQ \& WFQ

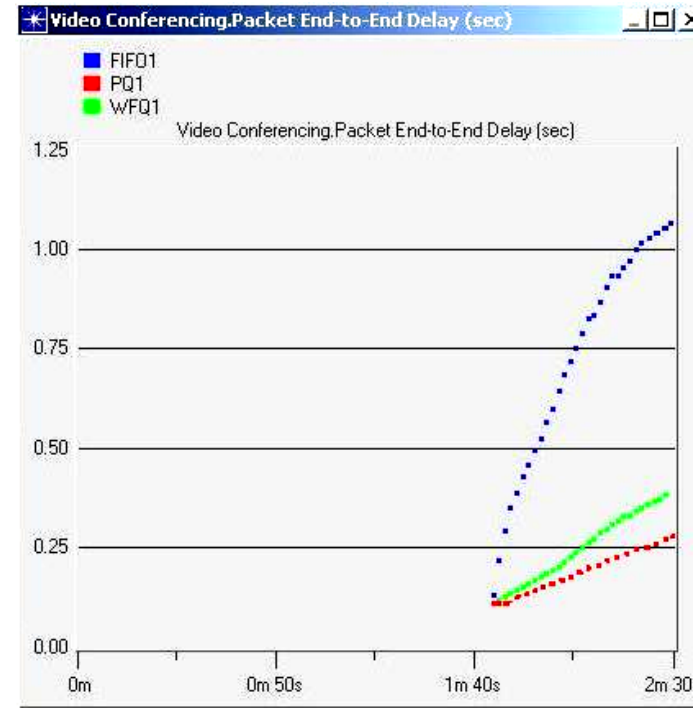

Fig. 12 (b) Delay variation for FIFO1, PQ1 \& WFQ1

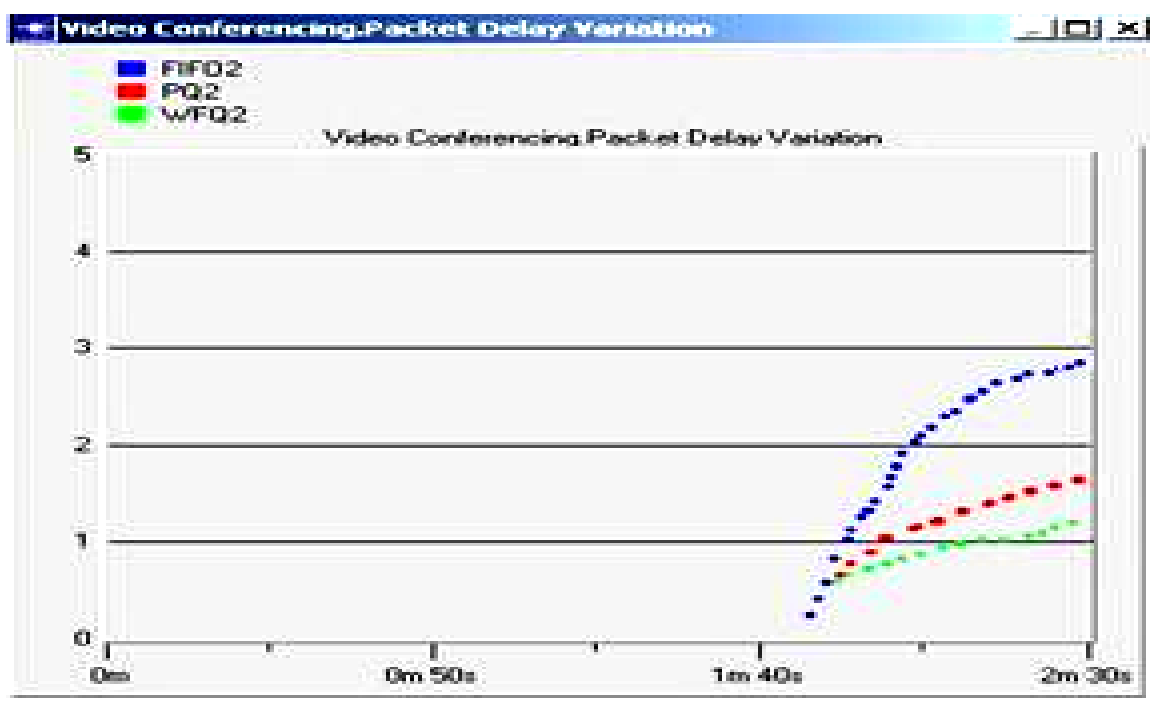

Fig. 12 (c) Delay variation for FIFO2, PQ2 and WFQ2

Figures (Fig.: 12a, 12b, 12c) shows Packet delay variation time for Video conference, where it can be observed that as the traffic increased the Packet delay variation time decreased for WFQ groups where as Packet delay variation time is increased for $\mathrm{PQ}$ groups. Like VoIP, FIFO group's Packet delay variation time is also always higher in Video conference.

\section{Conclusion}

After the comparison of the results it is clear that in case of PQ group the performance always depends on traffic's number and shows better output even when the number of clients increases and it's performance is better than FIFO group in some cases. FIFO group's performance is also better in some cases compared with PQ group.Now, if we compare WFQ group with FIFO and PQ group in case of traffic drop, File receiving, voice data receive and video conferencing; WFQ always shows the best performance among them. We know there are some problems with the integration between VOIP architecture and Internet. For any meaningful exchange, voice data communication must be a real time stream (you can't speak, wait for many seconds and then hear the other side answering). This is in contrast with the Internet architecture that is made of many routers, which can have a very high roundtrip 
time (RTT), this can delay the voice data in reaching the proper address at a continuous rate; so a few things have to be modified to get it properly working. As IP was designed for carrying data, so it does not provide real time Guarantees but only provides best effort service. For voice communications over IP to become acceptable to the users, the delay needs to be less than a threshold value and the IETF (Internet Engineering Task Force) is working on this aspect [9]. Now if considering results of WPQ algorithm can be applied to solve the time delay problem. Delays for applications like video conferencing are very apparent, causing the video signal to jerk and sputter [10]. This happened when FIFO algorithm used. Fair Queuing algorithm can solve the problem. But according to the simulation it is already proven that a modernized format of fair queuing WFQ (Weighted Fair Queue) can perform better. So ,it can be said with confidence that user traffic stream like voice, video, data can be easily transferred with it's efficient level performance by using Weighted Fair Queue algorithm in routers where the voice, video and data streams are routed to go to their desired destination.

\section{References}

[1] Davide Astuti , "Packet Handling", http://marco.uminho.pt/disciplinas/ST/packethandl ing.pdf .

[2] "FIFO (First in First out)",http://www.daxnetworks.com/Technology/T echDost/TD- 032206.pdf

[3] Hermawan Widiyanto, “ AWA

Network”http://awanetwork.blogspot.com/2007/09 /qos-priority-queueing.html

[4] RTS case study, "Get rid of traffic jam with priority queuing" http://www.rtsindia.com/pdf/PQ_CaseStudy\%20v er2.pdf

[5] Network World, "Weighted Fair Queuing” http://www.networkworld.com/details/659.html

[6] "Weighted Fair Queuing”
,http://en.wikipedia.org/wiki/Weighted_fair_queui ng

[7] E Aboelela, Morgan Kaufmann, "Network Simulation Experiments Manual”, 2003, ISBN: 0-12-042171-2

[8] Howard Van Horn, "Identifying and Integrating Component Collaboration Technologies (3)",http://faculty.capitolcollege.edu/Assinment\%202/IE715_Assignment2_ Student_Results.ppt

[9] Maheshwari, Harish, Mandhania, Sonali Sisodia "VoIP Technology: Overview and Enhancements" (MCA , I.I.P.S , D.A.V.V)

[10] Setrag Khoshafian, A. Brad Baker; "Contributor A. Brad Baker”, 1996, ISBN: 1558603123, 9781558603127

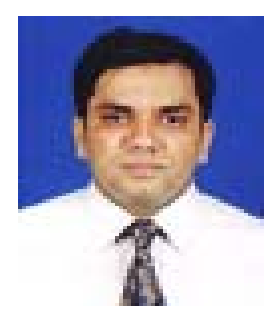

Mohammad Mirza Golam Rashed received his B.Sc and M.Sc in Physics from Jahangirnagar University, Savar, Dhaka in 1997 and 1998 respectively then he completed M.Sc in Telecommunication

Engineering and Computer Networks from London South Bank University of UK in July, 2005.Since January, 2007 he has been working as a Senior Lecturer at Daffodil International University. His fields of research interests include Wireless and Mobile Communication, Optical Fiber Communication and Computer Networking.

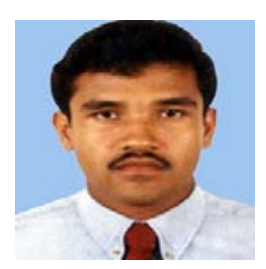

Md. Mamun Kabir received his B.Sc in Electronics and Telecom Engineering from Daffodil International University in 2009.Now; he is working as a Senior Hardware Engineer at Ryan's IT Limited, IDB Bhaban, Agargaon, Dhaka. His field of research interest is Wireless Network's Infrastructure and Security. 